\title{
Red Material
}

National Cancer Institute

\section{Source}

National Cancer Institute. Red Material. NCI Thesaurus. Code C124576.

Matter that is red in color. 